MATHEMATICS OF COMPUTATION

Volume 77, Number 261, January 2008, Pages 1-10

S 0025-5718(07)02037-6

Article electronically published on September 18, 2007

\title{
ANALYSIS OF A FINITE ELEMENT PML APPROXIMATION FOR THE THREE DIMENSIONAL TIME-HARMONIC MAXWELL PROBLEM
}

\author{
JAMES H. BRAMBLE AND JOSEPH E. PASCIAK
}

\begin{abstract}
In our paper [Math. Comp. 76, 2007, 597-614] we considered the acoustic and electromagnetic scattering problems in three spatial dimensions. In particular, we studied a perfectly matched layer (PML) approximation to an electromagnetic scattering problem. We demonstrated both the solvability of the continuous PML approximations and the exponential convergence of the resulting solution to the solution of the original acoustic or electromagnetic problem as the layer increased.

In this paper, we consider finite element approximation of the truncated PML electromagnetic scattering problem. Specifically, we consider approximations which result from the use of Nédélec (edge) finite elements. We show that the resulting finite element problem is stable and gives rise to quasi-optimal convergence when the mesh size is sufficiently small.
\end{abstract}

\section{INTRODUCTION}

The purpose of this paper is to analyze a finite element approximation of a problem which results when the time-harmonic electromagnetic scattering problem is approximated using a truncated PML approach. We shall demonstrate that the finite element method is stable and convergent provided that the grid size is sufficiently fine.

There are several aspects of the time-harmonic electromagnetic scattering problem which make it computationally challenging. First, the problem is not uniformly elliptic since the gradient part of the field is not controlled by the form. In addition, because of the time harmonic nature of the problem, the zeroth order term appears with a sign opposite to that of the curl-curl term. This causes the problem to have an indefinite character. The solvability of the continuous problem is, however, a consequence of the Silver-Müller radiation condition imposed at infinity. Unfortunately, the resulting solutions decay very slowly necessitating the use of either some type of artificial boundary condition or a boundary integral approach.

The so-called PML technique is perhaps one of the most effective of the artificial boundary condition approaches. This is because accurate solutions on a domain near the scatterer can be achieved by increasing the size of the computational region

Received by the editor September 11, 2006 and, in revised form, January 24, 2007.

2000 Mathematics Subject Classification. Primary 78M10, 65F10, 65N30.

Key words and phrases. Maxwell's equations, Helmholtz equation, time-harmonic acoustic and electromagnetic scattering, div-curl systems, PML layer.

This work was supported in part by the National Science Foundation through grant No. 0311902.

(C)2007 American Mathematical Society Reverts to public domain 28 years from publication 
by a factor of only around two. Moreover, implementation of the PML approach in a reasonably general code developed for the bounded domain problem is quite simple since it involves only a change in the coefficients.

Although there are many variants of the PML approach, we consider a version which coincides with a complex change of coordinates based on a spherical layer. This approach allows us to define a new problem (the infinite PML Problem) on the complement of the scatterer which satisfies:

(1) The solutions of the original and PML problems coincide on the interior of a ball of radius $r_{0}$.

(2) The solution of the infinite PML problem decays exponentially for large argument.

Because of the decay property, the solution of the infinite PML problem can be well approximated by a truncated (bounded domain) PML problem (see, [2, 3]). In particular, in [3] an inf-sup condition for the truncated domain problem is derived. This is a critical estimate for the finite element analysis which we provide here.

In this paper, we consider approximating the truncated PML problem using curlconforming finite elements $5,12,13$. Our analysis uses many of the ideas developed for curl-conforming finite element approximations to time-harmonic electromagnetic problems on bounded domains [7, 9, 10, 11]. We use a duality approach reminiscent of the argument of Schatz [14 with modifications to handle the lack of uniform ellipticity similar to those used in [6, 9].

The remainder of the paper is outlined as follows. In Section 2, we introduce the time-harmonic electromagnetic scattering problem and the infinite PML problem. Section 3 defines the truncated PML problem and its Galerkin approximation. The main result of the paper is stated and proved there.

\section{The SCATtERING PROBlem AND Infinite PML APPRoXimation}

Let $\Omega$ (the scatterer) be a bounded domain in $\mathbb{R}^{3}$ with polyhedral boundary. We will consider the time-harmonic electromagnetic scattering problem. We seek vector fields $\boldsymbol{E}$ and $\boldsymbol{H}$ defined on the complement $\Omega^{c}$ satisfying

$$
\begin{aligned}
-i k \mu \boldsymbol{H}+\boldsymbol{\nabla} \times \boldsymbol{E} & =\mathbf{0}, \text { in } \Omega^{c}, \\
-i k \epsilon \boldsymbol{E}-\boldsymbol{\nabla} \times \boldsymbol{H} & =\mathbf{0}, \text { in } \Omega^{c}, \\
\boldsymbol{n} \times \boldsymbol{E} & =\boldsymbol{n} \times \boldsymbol{g}, \text { on } \partial \Omega, \\
\lim _{\rho \rightarrow \infty} \rho(\mu \boldsymbol{H} \times \hat{\boldsymbol{x}}-\boldsymbol{E}) & =\mathbf{0} .
\end{aligned}
$$

Here $\boldsymbol{g}$ results from a given incidence field, $\mu$ is the magnetic permeability, $\epsilon$ is the electric permittivity, $k$ is the wave number, and $\boldsymbol{n}$ is the outward unit normal on $\partial \Omega$. Also $\hat{\boldsymbol{x}}$ is a unit vector in the direction of $\boldsymbol{x}$ and $\rho=|\boldsymbol{x}|$. The last line corresponds to the Silver-Müller condition at infinity. We assume that the coefficients $\mu$ and $\epsilon$ are real and positive, bounded away from zero and constant outside of some ball (of radius $r_{0}$ ).

We note that $\Omega^{c}$ need not be simply connected and we do not assume that its boundary is simply connected. We let $\Gamma_{j}, j=1, \ldots, p$ denote the connected components of $\partial \Omega^{c}$.

We introduce some notation that will be used in the remainder of the paper. For a domain $D$, let $L^{2}(D)$ be the space of (complex valued) square integrable functions on $D$ and $\boldsymbol{L}^{2}(D)=\left(L^{2}(D)\right)^{3}$ be the space of vector valued $L^{2}$-functions. We shall 
use $(\cdot, \cdot)_{\Omega}$ to denote the (vector or scalar Hermitian) $L^{2}(\Omega)$ inner product and $\langle\cdot, \cdot\rangle_{\Gamma}$ to denote the (vector or scalar Hermitian) $L^{2}(\Gamma)$ boundary inner product. For a real number $s$, the scalar and vector Sobolev spaces on $D$ will be denoted $H^{s}(D)$ and $\boldsymbol{H}^{s}(D)$ respectively. Let $\boldsymbol{H}(\mathbf{c u r l} ; D)$ be the set of vector valued functions which, along with their curls, are in $\boldsymbol{L}^{2}(D) . \boldsymbol{H}_{0}(\mathbf{c u r l} ; D)$ denotes the functions $\boldsymbol{f}$ in $\boldsymbol{H}(\mathbf{c u r l} ; D)$ satisfying $\boldsymbol{n} \times \boldsymbol{f}=\mathbf{0}$ on $\partial \Omega$. We assume that $\boldsymbol{n} \times \boldsymbol{g}$ above is the trace $\boldsymbol{n} \times \widehat{\boldsymbol{g}}$ of a function $\widehat{\boldsymbol{g}} \in \boldsymbol{H}\left(\mathbf{c u r l} ; \Omega^{c}\right)$ supported close to $\partial \Omega$.

For a subdomain $D$ contained in our computational domain $\Omega_{\infty}$, by extension by zero, we identify $H_{0}^{1}(D)$ with

$$
\left\{v \in H_{0}^{1}\left(\Omega^{c}\right): \operatorname{supp}(v) \subseteq \bar{D}\right\}
$$

and $\boldsymbol{H}_{0}(\mathbf{c u r l} ; D)$ with

$$
\left.\left\{\boldsymbol{v} \in \boldsymbol{H}_{0}\left(\operatorname{curl} ; \Omega^{c}\right)\right): \operatorname{supp}(\boldsymbol{v}) \subseteq \bar{D}\right\} .
$$

For convenience, we shall take $\mu=\epsilon=1$ in (2.1) as all of our results extend to the more general case as long as the coefficients are constant outside of a ball of radius $r_{0}$. We can reduce (2.1) to a single equation involving $\boldsymbol{E}$ by eliminating $\boldsymbol{H}$. This gives

$$
\begin{aligned}
&-\nabla \times \nabla \times \boldsymbol{E}+k^{2} \boldsymbol{E}=\mathbf{0} \text { in } \Omega^{c}, \\
& \boldsymbol{n} \times \boldsymbol{E}=\boldsymbol{n} \times \boldsymbol{g} \text { on } \partial \Omega, \\
& \lim _{\rho \rightarrow \infty} \rho((\boldsymbol{\nabla} \times \boldsymbol{E}) \times \hat{\boldsymbol{x}}-i k \boldsymbol{E})=\mathbf{0} .
\end{aligned}
$$

As in [3, we introduce bounded subdomains of $\Omega^{c}$ with spherical outer boundaries. Let $0<r_{0}<r_{1}<r_{2}$ be real numbers and let $\Omega_{i}$ denote (the interior of) the open ball $B_{i}$ of radius $r_{i}$ excluding $\bar{\Omega}$. We assume that $r_{0}$ is large enough so that the corresponding ball contains $\bar{\Omega}$ and that the origin is contained in $\Omega$. We denote the outer boundary of $\Omega_{i}$ by $\Gamma_{i}$. The domain $\Omega_{2}$ is contained in our computational domain which we denote by $\Omega_{\infty}$. Here $\Omega_{\infty}$ is a bounded subset of $\Omega^{c}$ with a polyhedral boundary, part of which is $\partial \Omega$. The outer boundary of $\Omega_{\infty}$ is denoted by $\Gamma_{\infty}$

As discussed in 4, the PML problem can be viewed as a complex coordinate transformation. Following [8, a transitional layer based on spherical geometry is defined which results in a constant coefficient problem outside the transition. Given $\sigma_{0}, r_{1}$, and $r_{2}$, we start with a function $\widetilde{\sigma} \in C^{2}\left(\mathbb{R}^{+}\right)$satisfying

$$
\begin{aligned}
& \widetilde{\sigma}(\rho)=0, \quad \text { for } 0 \leq \rho \leq r_{1}, \\
& \widetilde{\sigma}(\rho)=\sigma_{0}, \quad \text { for } \rho \geq r_{2}, \\
& \widetilde{\sigma}(\rho) \text { increasing for } \rho \in\left(r_{1}, r_{2}\right) .
\end{aligned}
$$

We define

$$
\tilde{\rho}=\rho(1+i \widetilde{\sigma}) \equiv \rho \widetilde{d} .
$$

One obvious construction of such a function $\widetilde{\sigma}$ in the transition layer $r_{1} \leq \rho \leq r_{2}$ with the above properties is given by the fifth degree polynomial,

$\tilde{\sigma}(\rho)=\sigma_{0}\left(\int_{r_{1}}^{\rho}\left(t-r_{1}\right)^{2}\left(r_{2}-t\right)^{2} d t\right)\left(\int_{r_{1}}^{r_{2}}\left(t-r_{1}\right)^{2}\left(r_{2}-t\right)^{2} d t\right)^{-1}, \quad$ for $r_{1} \leq \rho \leq r_{2}$.

A smoother $\widetilde{\sigma}$ can be constructed by increasing the exponents in the above formula. 
Each component, $u$, of the solution $\boldsymbol{E}$ of (2.2) satisfies the Helmholtz equation with Sommerfeld radiation condition, i.e.,

$$
\begin{aligned}
\Delta u+k^{2} u & =0, \quad \text { for } \rho>r_{0}, \\
\lim _{\rho \rightarrow \infty} \rho(\nabla u \cdot \hat{\boldsymbol{x}}-i k u) & =0 .
\end{aligned}
$$

It follows that the solution of (2.2) can be expanded

$$
\boldsymbol{E}=\sum_{n=0}^{\infty} \sum_{m=-n}^{n} \boldsymbol{a}_{n, m} h_{n}^{1}(k \rho) Y_{n}^{m}(\theta, \phi), \quad \text { for } \rho \geq r_{0} .
$$

Here $h_{n}^{1}(r)$ are spherical Bessel functions of the third kind (Hankel functions), $Y_{n}^{m}$ are spherical harmonics (see, e.g., [10] for details) and $\boldsymbol{a}_{n, m}$ are vector valued constants.

The (infinite domain) PML solution is defined by

$$
\widetilde{\boldsymbol{E}}=\left\{\begin{array}{l}
\boldsymbol{E}(\boldsymbol{x}), \quad \text { for }|\boldsymbol{x}| \leq r_{1}, \\
\sum_{n=1}^{\infty} \sum_{m=-n}^{n} \boldsymbol{a}_{n, m} h_{n}^{1}(k \tilde{\rho}) Y_{n}^{m}(\theta, \phi), \quad \text { for } \rho=|\boldsymbol{x}| \geq r_{1} .
\end{array}\right.
$$

By construction $\widetilde{\boldsymbol{E}}$ and $\boldsymbol{E}$ coincide on $\Omega_{1}$. Furthermore, the complex shift in the argument of $h_{n}^{1}$ above $(k \rho$ replaced by $k \tilde{\rho})$ guarantees exponential decay of $\widetilde{\boldsymbol{E}}$ as $\rho$ tends to infinity.

The PML solution defined above satisfies a differential equation involving $\tilde{\rho}$ and $\frac{d \tilde{\rho}}{d \rho}$. A simple computation shows that

$$
\frac{d \tilde{\rho}}{d \rho}=(1+i \sigma(\rho)) \equiv d
$$

where

$$
\sigma(\rho)=\tilde{\sigma}(\rho)+\rho \widetilde{\sigma}^{\prime}(\rho) .
$$

It follows that $\sigma$ is in $C^{1}\left(\mathbb{R}^{+}\right)$and satisfies

$$
\begin{aligned}
& \sigma(\rho)=0, \quad \text { for } 0 \leq \rho \leq r_{1}, \\
& \sigma(\rho)>\widetilde{\sigma}(\rho), \quad \text { for } \rho \in\left(r_{1} r_{2}\right), \\
& \sigma(\rho)=\sigma_{0}, \quad \text { for } \rho \geq r_{2} .
\end{aligned}
$$

The solution $\widetilde{\boldsymbol{E}}$ satisfies Maxwell's equations using the spherical coordinates $(\tilde{\rho}, \theta, \phi)[10]$. More precisely,

$$
\begin{aligned}
&-\widetilde{\nabla} \times \widetilde{\nabla} \times \widetilde{\boldsymbol{E}}+k^{2} \widetilde{\boldsymbol{E}}=\mathbf{0} \text { in } \Omega^{c}, \\
& \boldsymbol{n} \times \widetilde{\boldsymbol{E}}=\boldsymbol{n} \times \boldsymbol{g} \text { on } \partial \Omega, \\
& \widetilde{\boldsymbol{E}} \text { bounded at } \infty .
\end{aligned}
$$

For $\widetilde{\boldsymbol{E}}$ expanded in spherical coordinates,

$$
\widetilde{\boldsymbol{E}}=\widetilde{\boldsymbol{E}}_{\rho} \boldsymbol{e}_{\rho}+\widetilde{\boldsymbol{E}}_{\theta} \boldsymbol{e}_{\theta}+\widetilde{\boldsymbol{E}}_{\phi} \boldsymbol{e}_{\phi},
$$


we have

$$
\begin{aligned}
\widetilde{\boldsymbol{\nabla}} \times \widetilde{\boldsymbol{E}} & =\frac{1}{\widetilde{d} \rho \sin \theta}\left(\frac{\partial}{\partial \theta}\left(\sin \theta \widetilde{\boldsymbol{E}}_{\phi}\right)-\frac{\partial \widetilde{\boldsymbol{E}}_{\theta}}{\partial \phi}\right) \boldsymbol{e}_{\rho} \\
& +\frac{1}{\widetilde{d} \rho}\left(\frac{1}{\sin \theta} \frac{\partial \widetilde{\boldsymbol{E}}_{\rho}}{\partial \phi}-\frac{1}{d} \frac{\partial}{\partial \rho}\left(\widetilde{d} \rho \widetilde{\boldsymbol{E}}_{\phi}\right)\right) \boldsymbol{e}_{\theta} \\
& +\frac{1}{\widetilde{d} \rho}\left(\frac{1}{d} \frac{\partial}{\partial \rho}\left(\widetilde{d} \rho \widetilde{\boldsymbol{E}}_{\theta}\right)-\frac{\partial \widetilde{\boldsymbol{E}}_{\rho}}{\partial \theta}\right) \boldsymbol{e}_{\phi} .
\end{aligned}
$$

\section{The truncated PML problem and its Galerkin approximation}

Since the solution of (2.5) coincides with that of (2.2) on $\Omega_{1}$ while rapidly decaying as $\rho$ tends to infinity, it is natural to truncate to a finite computational domain $\Omega_{\infty}$ and impose convenient boundary conditions on the outer boundary $\Gamma_{\infty}$. We shall always require that the transitional region is contained in $\Omega_{\infty}$, i.e., $\bar{\Omega}_{2} \subset \Omega_{\infty}$.

The truncated PML approximation is then given as the vector function $\widetilde{\boldsymbol{E}_{\boldsymbol{t}}}$ defined on $\Omega_{\infty}$ satisfying

$$
\begin{aligned}
-\widetilde{\nabla} \times \widetilde{\nabla} \times \widetilde{\boldsymbol{E}_{\boldsymbol{t}}}+k^{2} \widetilde{\boldsymbol{E}_{\boldsymbol{t}}} & =\mathbf{0} \text { in } \Omega_{\infty}, \\
\boldsymbol{n} \times \widetilde{\boldsymbol{E}_{\boldsymbol{t}}} & =\boldsymbol{n} \times \boldsymbol{g} \text { on } \partial \Omega, \\
\boldsymbol{n} \times \widetilde{\boldsymbol{E}_{\boldsymbol{t}}} & =\mathbf{0} \text { on } \Gamma_{\infty} .
\end{aligned}
$$

We shall assume that $\Omega_{\infty}$ is sufficiently large so that we can apply the results of [3] to conclude existence and uniqueness of solutions of (3.1).

We can write the derivatives in (3.1) in terms of cartesian coordinates. Following [10], we define the diagonal matrices (in spherical coordinates)

$$
\boldsymbol{A} \boldsymbol{v}=\widetilde{d}^{-2} v_{\rho} \boldsymbol{e}_{\rho}+(\widetilde{d} d)^{-1}\left(v_{\theta} \boldsymbol{e}_{\theta}+v_{\phi} \boldsymbol{e}_{\phi}\right)
$$

and

$$
\boldsymbol{B} \boldsymbol{v}=d v_{\rho} \boldsymbol{e}_{\rho}+\widetilde{d}\left(v_{\theta} \boldsymbol{e}_{\theta}+v_{\phi} \boldsymbol{e}_{\phi}\right) .
$$

Then, $\widetilde{\boldsymbol{\nabla}} \times \widetilde{\boldsymbol{E}}=\boldsymbol{A} \boldsymbol{\nabla} \times(\boldsymbol{B} \widetilde{\boldsymbol{E}})$. The matrices $\boldsymbol{A}$ and $\boldsymbol{B}$ have simple representations in cartesian coordinates, e.g.,

$$
\boldsymbol{A}=(\widetilde{d} d)^{-1} \boldsymbol{I}+\frac{\widetilde{d}^{-2}-(\widetilde{d} d)^{-1}}{\rho^{2}}\left(\begin{array}{ccc}
x_{1}^{2} & x_{1} x_{2} & x_{1} x_{3} \\
x_{2} x_{1} & x_{2}^{2} & x_{2} x_{3} \\
x_{3} x_{1} & x_{3} x_{2} & x_{3}^{2}
\end{array}\right)
$$

Here $\boldsymbol{I}$ denotes the $3 \times 3$ identity matrix.

Following [10, we first define a weak form of the PML problem (3.1) by setting $\widetilde{\boldsymbol{E}_{\boldsymbol{t}}}=\widehat{\boldsymbol{g}}-\boldsymbol{w}$ and setting up a variational problem for $\boldsymbol{\Xi}=\boldsymbol{B} \boldsymbol{w} \in \boldsymbol{H}_{0}\left(\mathbf{c u r l} ; \Omega_{\infty}\right)$, i.e.,

$$
\mathcal{A}(\boldsymbol{\Xi}, \boldsymbol{\Psi})=\mathcal{A}(\boldsymbol{B} \widehat{\boldsymbol{g}}, \boldsymbol{\Psi}), \quad \text { for all } \boldsymbol{\Psi} \in \boldsymbol{H}_{0}\left(\operatorname{curl} ; \Omega_{\infty}\right) .
$$

Here the sesquilinear form $\mathcal{A}$ is given by

$$
\mathcal{A}(\boldsymbol{\Theta}, \boldsymbol{\Psi}) \equiv\left(\boldsymbol{\mu}^{-1} \boldsymbol{\nabla} \times \boldsymbol{\Theta}, \boldsymbol{\nabla} \times \boldsymbol{\Psi}\right)-k^{2}(\boldsymbol{\mu} \boldsymbol{\Theta}, \boldsymbol{\Psi}), \quad \text { for all } \boldsymbol{\Theta}, \boldsymbol{\Psi} \in \boldsymbol{H}\left(\operatorname{curl} ; \Omega^{c}\right)
$$

and $\boldsymbol{\mu}=(\boldsymbol{A} \boldsymbol{B})^{-1}$. For convenience, we have used the notation $(\cdot, \cdot)$ above to denote the inner product on $\Omega_{\infty}$, as inner products on $\Omega_{\infty}$ will be used extensively in the remainder of the paper. 
We consider the Nédélec finite element approximation to problem (3.2) which we restate as

$$
\mathcal{A}(\boldsymbol{\Xi}, \Psi)=\boldsymbol{F}(\boldsymbol{\Psi}) \quad \text { for all } \boldsymbol{\Psi} \in \boldsymbol{H}_{0}\left(\operatorname{curl} ; \Omega_{\infty}\right) .
$$

Here $\boldsymbol{F}$ denotes the functional corresponding to the right hand side of (3.2).

For convenience, let $Q_{h}$ denote the curl conforming approximation subspace of Nédélec elements on tetrahedra shaped elements consisting of piecewise polynomials of maximum degree $r$ [5, 10, 12, 13. Our results extend to the brick shaped elements. The finite element approximation to (3.3) is: Find $\boldsymbol{\Xi}_{h} \in Q_{h}$ satisfying

$$
\mathcal{A}\left(\boldsymbol{\Xi}_{h}, \boldsymbol{\Phi}\right)=\boldsymbol{F}(\boldsymbol{\Phi}) \text { for all } \boldsymbol{\Phi} \in Q_{h} .
$$

The main result of this paper is contained in the following theorem. Its proof will be given in the remainder of this section.

Theorem 3.1. There exists $h_{0}>0$ such that if $h \leq h_{0}$, problem (3.4) has a unique solution $\boldsymbol{\Xi}_{h}$ which satisfies

$$
\left\|\boldsymbol{\Xi}_{h}-\boldsymbol{\Xi}\right\|_{\boldsymbol{H}\left(\operatorname{curl} ; \Omega_{\infty}\right)} \leq C \inf _{\boldsymbol{V} \in Q_{h}}\|\boldsymbol{V}-\boldsymbol{\Xi}\|_{\boldsymbol{H}\left(\operatorname{curl} ; \Omega_{\infty}\right)} .
$$

The proof of the above theorem is based on a perturbation argument and duality. Such arguments have been applied to uniformly elliptic problems [14] as well as time harmonic Maxwell problems on bounded domains [9, 10.

In spherical coordinates, the matrix function $\boldsymbol{\mu}$ is represented by multiplication by a diagonal matrix with complex diagonal entries $\left\{D_{11}, D_{22}, D_{33}\right\} \equiv\left\{\widetilde{d}^{2} / d, d, d\right\}$. Because of the assumptions on $\widetilde{\sigma}$ it follows that there are constants $c_{0}, c_{1}$ satisfying

$$
c_{0} \leq \operatorname{Re}\left(D_{j j}\right) \leq c_{1} \text { and } c_{0} \leq \operatorname{Re}\left(D_{j j}^{-1}\right) \leq c_{1} .
$$

Setting

$$
\hat{\mathcal{A}}(\boldsymbol{W}, \boldsymbol{V})=k^{2}(\boldsymbol{\mu} \boldsymbol{W}, \boldsymbol{V})+\left(\boldsymbol{\mu}^{-1} \nabla \times \boldsymbol{W}, \boldsymbol{\nabla} \times \boldsymbol{V}\right)
$$

for $\boldsymbol{W}, \boldsymbol{V} \in \boldsymbol{H}\left(\operatorname{curl} ; \Omega_{\infty}\right)$, it follows that

and

$$
\|\boldsymbol{W}\|_{\boldsymbol{H}\left(\operatorname{curl} ; \Omega_{\infty}\right)}^{2} \leq C R e(\hat{\mathcal{A}}(\boldsymbol{W}, \boldsymbol{W}))
$$

We will first prove the inf-sup condition corresponding to problem (3.2), namely that for $\boldsymbol{V} \in \boldsymbol{H}_{0}\left(\operatorname{curl} ; \Omega_{\infty}\right)$,

$$
\|\boldsymbol{V}\|_{\boldsymbol{H}\left(\mathbf{c u r l} ; \Omega_{\infty}\right)} \leq C \sup _{\boldsymbol{\Theta} \in \boldsymbol{H}_{0}\left(\operatorname{curl} ; \Omega_{\infty}\right)} \frac{|\mathcal{A}(\boldsymbol{V}, \boldsymbol{\Theta})|}{\|\boldsymbol{\Theta}\|_{\boldsymbol{H}\left(\operatorname{curl} ; \Omega_{\infty}\right)}}
$$

This will be a consequence of an inf-sup condition proved in 3 .

We follow [3] and set

$$
\boldsymbol{X}_{N}\left(\Omega_{\infty}\right)=\boldsymbol{H}_{0}\left(\operatorname{curl} ; \Omega_{\infty}\right) \cap \boldsymbol{H}^{0}\left(\operatorname{div} ; \boldsymbol{\mu}, \Omega_{\infty}\right),
$$

where $\boldsymbol{H}^{0}\left(\operatorname{div} ; \boldsymbol{\mu}, \Omega_{\infty}\right)=\left\{\boldsymbol{U} \in \boldsymbol{L}^{2}\left(\Omega_{\infty}\right): \nabla \cdot(\boldsymbol{\mu} \boldsymbol{U})=0\right\}$. It was shown in 3 that for $\boldsymbol{v} \in \boldsymbol{X}_{N}\left(\Omega_{\infty}\right)$,

$$
\|\boldsymbol{v}\|_{\boldsymbol{H}\left(\mathbf{\operatorname { c u r l } ; \Omega _ { \infty } )} \leq\right.} \leq C \sup _{\boldsymbol{\phi} \in \boldsymbol{X}_{N}\left(\Omega_{\infty}\right)} \frac{|\mathcal{A}(\boldsymbol{v}, \boldsymbol{\phi})|}{\|\boldsymbol{\phi}\|_{\boldsymbol{H}\left(\mathbf{c u r l} ; \Omega_{\infty}\right)}} .
$$

Let $\boldsymbol{V}$ be in $\boldsymbol{H}_{0}\left(\operatorname{curl} ; \Omega_{\infty}\right)$ and set $\boldsymbol{V}=\boldsymbol{v}+\nabla \psi$ where $\psi \in H_{0}^{1}\left(\Omega_{\infty}\right)$ solves

$$
(\boldsymbol{\mu} \nabla \psi, \nabla \theta)=(\boldsymbol{\mu} \boldsymbol{V}, \boldsymbol{\nabla} \theta), \quad \text { for all } \theta \in H_{0}^{1}\left(\Omega_{\infty}\right),
$$


so that $\boldsymbol{v}$ is in $\boldsymbol{X}_{N}\left(\Omega_{\infty}\right)$. Thus,

$$
\begin{aligned}
\|\boldsymbol{v}\|_{\boldsymbol{H}\left(\mathbf{c u r l} ; \Omega_{\infty}\right)} & \leq C \sup _{\boldsymbol{\phi} \in \boldsymbol{X}\left(\Omega_{\infty}\right)} \frac{|\mathcal{A}(\boldsymbol{V}, \boldsymbol{\phi})|}{\|\boldsymbol{\phi}\|_{\boldsymbol{H}\left(\mathbf{c u r l} ; \Omega_{\infty}\right)}}+C\|\nabla \psi\|_{\boldsymbol{L}^{2}\left(\Omega_{\infty}\right)} \\
& \leq C \sup _{\boldsymbol{\Theta} \in \boldsymbol{H}_{0}\left(\mathbf{c u r l} ; \Omega_{\infty}\right)} \frac{|\mathcal{A}(\boldsymbol{V}, \boldsymbol{\Theta})|}{\|\boldsymbol{\Theta}\|_{\boldsymbol{H}\left(\mathbf{c u r l} ; \Omega_{\infty}\right)}}+C\|\nabla \psi\|_{\boldsymbol{L}^{2}\left(\Omega_{\infty}\right)} .
\end{aligned}
$$

Now

$$
\|\nabla \psi\|_{\boldsymbol{L}^{2}\left(\Omega_{\infty}\right)}^{2} \leq C|\mathcal{A}(\nabla \psi, \nabla \psi)|=C|\mathcal{A}(\boldsymbol{V}, \nabla \psi)|,
$$

from which it follows easily that

$$
\|\nabla \psi\|_{\boldsymbol{H}\left(\mathbf{\operatorname { c u r l } ; \Omega _ { \infty } )}\right.}=\|\nabla \psi\|_{L^{2}\left(\Omega_{\infty}\right)} \leq C \sup _{\boldsymbol{\Theta} \in \boldsymbol{H}_{0}\left(\operatorname{curl} ; \Omega_{\infty}\right)} \frac{|\mathcal{A}(\boldsymbol{V}, \boldsymbol{\Theta})|}{\|\boldsymbol{\Theta}\|_{\boldsymbol{H}\left(\mathbf{c u r l} ; \Omega_{\infty}\right)}} .
$$

The inf-sup condition (3.8) then follows from the triangle inequality, (3.10) and (3.11).

Because of (3.6), we can define a Galerkin projector: $\hat{P}_{h}: \boldsymbol{H}\left(\mathbf{c u r l} ; \Omega_{\infty}\right) \rightarrow Q_{h}$ satisfying

$$
\hat{\mathcal{A}}\left(\boldsymbol{\Phi}, \hat{P}_{h} \boldsymbol{V}\right)=\hat{\mathcal{A}}(\boldsymbol{\Phi}, \boldsymbol{V}), \quad \text { for all } \boldsymbol{\Phi} \in Q_{h} .
$$

Clearly, $\hat{P}_{h}$ is bounded and gives rise to quasi-optimal convergence. We then have the following lemma which we shall prove at the end of this section.

Lemma 3.1. Let $\hat{P}_{h}$ be defined by (3.12). Then, for $\boldsymbol{V}_{h} \in Q_{h}, \boldsymbol{\Theta} \in \boldsymbol{H}_{0}\left(\operatorname{curl} ; \Omega_{\infty}\right)$, and for some $s>1 / 2$,

$$
\left|\left(\boldsymbol{\mu} \boldsymbol{V}_{h}, \boldsymbol{\Theta}-\hat{P}_{h} \boldsymbol{\Theta}\right)\right| \leq C h^{s}\|\boldsymbol{\Theta}\|_{\boldsymbol{H}\left(\operatorname{curl} ; \Omega_{\infty}\right)}\left\|\boldsymbol{V}_{h}\right\|_{\boldsymbol{H}\left(\operatorname{curl} ; \Omega_{\infty}\right)} .
$$

Using the above lemma, we can now prove Theorem 3.1 .

Proof of Theorem 3.1. It is clear that the theorem will follow if we verify the discrete inf-sup condition: For $\boldsymbol{V}_{h} \in Q_{h}$,

$$
\left\|\boldsymbol{V}_{h}\right\|_{\boldsymbol{H}\left(\mathbf{c u r l} ; \Omega_{\infty}\right)} \leq C \sup _{\boldsymbol{\Theta} \in Q_{h}} \frac{\left|\mathcal{A}\left(\boldsymbol{V}_{h}, \boldsymbol{\Theta}\right)\right|}{\|\boldsymbol{\Theta}\|_{\boldsymbol{H}\left(\mathbf{c u r l} ; \Omega_{\infty}\right)}} .
$$

Applying (3.8) with $\boldsymbol{V}=\boldsymbol{V}_{h} \in Q_{h}$, we have

$$
\begin{aligned}
C\left\|\boldsymbol{V}_{h}\right\|_{\boldsymbol{H}\left(\operatorname{curl} ; \Omega_{\infty}\right)} \leq \sup _{\boldsymbol{\Theta} \in \boldsymbol{H}_{0}\left(\operatorname{curl} ; \Omega_{\infty}\right)} \frac{\left|\mathcal{A}\left(\boldsymbol{V}_{h}, \boldsymbol{\Theta}\right)\right|}{\|\boldsymbol{\Theta}\|_{\boldsymbol{H}\left(\mathbf{c u r l} ; \Omega_{\infty}\right)}} \\
\quad \leq \sup _{\boldsymbol{\Theta} \in \boldsymbol{H}_{0}\left(\operatorname{curl} ; \Omega_{\infty}\right)} \frac{\left|\mathcal{A}\left(\boldsymbol{V}_{h}, \boldsymbol{\Theta}-\hat{P}_{h} \boldsymbol{\Theta}\right)\right|}{\|\boldsymbol{\Theta}\|_{\boldsymbol{H}\left(\mathbf{c u r l} ; \Omega_{\infty}\right)}}+\sup _{\boldsymbol{\Theta} \in \boldsymbol{H}_{0}\left(\operatorname{curl} ; \Omega_{\infty}\right)} \frac{\left|\mathcal{A}\left(\boldsymbol{V}_{h}, \hat{P}_{h} \boldsymbol{\Theta}\right)\right|}{\|\boldsymbol{\Theta}\|_{\boldsymbol{H}\left(\mathbf{c u r l} ; \Omega_{\infty}\right)}} \\
\quad=\sup _{\boldsymbol{\Theta} \in \boldsymbol{H}_{0}\left(\operatorname{curl} ; \Omega_{\infty}\right)} \frac{2 k^{2}\left|\left(\boldsymbol{\mu} \boldsymbol{V}_{h}, \boldsymbol{\Theta}-\hat{P}_{h} \boldsymbol{\Theta}\right)\right|}{\|\boldsymbol{\Theta}\|_{\boldsymbol{H}\left(\mathbf{c u r l} ; \Omega_{\infty}\right)}}+\sup _{\boldsymbol{\Theta} \in \boldsymbol{H}_{0}\left(\operatorname{curl} ; \Omega_{\infty}\right)} \frac{\left|\mathcal{A}\left(\boldsymbol{V}_{h}, \hat{P}_{h} \boldsymbol{\Theta}\right)\right|}{\|\boldsymbol{\Theta}\|_{\boldsymbol{H}\left(\mathbf{c u r l} ; \Omega_{\infty}\right)}} .
\end{aligned}
$$

Applying the lemma and stability of $\hat{P}_{h}$ in $\boldsymbol{H}_{0}\left(\mathbf{c u r l} ; \Omega_{\infty}\right)$, we have that

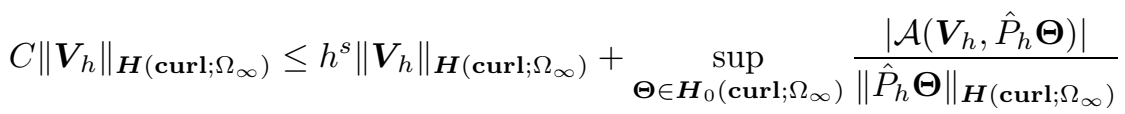

$$
\begin{aligned}
& =h^{s}\left\|\boldsymbol{V}_{h}\right\|_{\boldsymbol{H}\left(\operatorname{curl} ; \Omega_{\infty}\right)}+\sup _{\boldsymbol{\Theta}_{h} \in Q_{h}} \frac{\left|\mathcal{A}\left(\boldsymbol{V}_{h}, \boldsymbol{\Theta}_{h}\right)\right|}{\left\|\boldsymbol{\Theta}_{h}\right\|_{\boldsymbol{H}\left(\mathbf{c u r l} ; \Omega_{\infty}\right)}} .
\end{aligned}
$$

Thus, (3.14) follows for $h$ sufficiently small. 
We need an additional result for the proof of the lemma. To this end, we introduce

$$
\boldsymbol{X}_{T}\left(\Omega_{\infty}\right)=\boldsymbol{H}\left(\operatorname{curl} ; \Omega_{\infty}\right) \cap \boldsymbol{H}_{0}^{0}\left(\operatorname{div} ; \boldsymbol{\mu}, \Omega_{\infty}\right),
$$

where $\boldsymbol{H}_{0}^{0}\left(\operatorname{div} ; \boldsymbol{\mu}, \Omega_{\infty}\right)=\left\{\boldsymbol{U} \in \boldsymbol{H}_{0}\left(\operatorname{div} ; \Omega_{\infty}\right): \nabla \cdot(\boldsymbol{\mu} \boldsymbol{U})=0\right\}$. The following proposition was proved in [3] for $X_{N}\left(\Omega_{\infty}\right)$. The proof for $\boldsymbol{X}_{T}\left(\Omega_{\infty}\right)$ is similar.

Proposition 3.1. $\boldsymbol{X}_{N}\left(\Omega_{\infty}\right)$ and $\boldsymbol{X}_{T}\left(\Omega_{\infty}\right)$ are contained in $\boldsymbol{H}^{s}\left(\Omega_{\infty}\right)$ for some $s>$ 1/2. Moreover,

$$
\|\boldsymbol{V}\|_{\boldsymbol{H}^{s}\left(\Omega_{\infty}\right)} \leq C\|\boldsymbol{V}\|_{\boldsymbol{H}\left(\mathbf{c u r l} ; \Omega_{\infty}\right)}, \quad \text { for all } \boldsymbol{V} \in \boldsymbol{X}_{N}\left(\Omega_{\infty}\right) \cup \boldsymbol{X}_{T}\left(\Omega_{\infty}\right) .
$$

We will now prove the lemma.

Proof of Lemma 3.1. For $i=1, \ldots, p$, define $\hat{\psi}_{i}$ to be the function in $H^{1}\left(\Omega_{\infty}\right)$ satisfying

$$
\begin{aligned}
& -\Delta \hat{\psi}_{i}=0, \quad \text { in } \Omega_{\infty}, \\
& \hat{\psi}_{i}=0, \quad \text { on } \Gamma_{j}(j=1, \ldots, p, \infty, j \neq i), \\
& \hat{\psi}_{i}=1, \quad \text { on } \Gamma_{i} \text {. }
\end{aligned}
$$

Let $W_{d}$ be the $p$-dimensional space spanned by $\left\{\hat{\psi}_{i}\right\}$ for $i=1, \ldots, p$ and set $\tilde{H}_{0}^{1}\left(\Omega_{\infty}\right)=H_{0}^{1}\left(\Omega_{\infty}\right)+W_{d}$. It is an easy consequence of Theorem 3.17 and Corollary 3.16 of [1] that the sequence

$$
\widetilde{H}_{0}^{1}\left(\Omega_{\infty}\right) \stackrel{\nabla}{\longrightarrow} \boldsymbol{H}_{0}\left(\operatorname{curl} ; \Omega_{\infty}\right) \stackrel{\nabla \times}{\longrightarrow} \boldsymbol{H}_{0}\left(\operatorname{div} ; \Omega_{\infty}\right)
$$

is exact.

On the discrete level, we introduce the space $\tilde{S}_{h}$ which is defined to be the $H^{1}\left(\Omega_{\infty}\right)$ conforming space consisting of functions which are piecewise polynomials of degree $r$, are constant on $\Gamma_{i}, i=1, \ldots, p$ and vanish on $\Gamma_{\infty}$. It is an easy consequence of Lemma 5.3 (Chapter 3) of [5] that if $\boldsymbol{q} \in Q_{h}$ satisfies $\boldsymbol{\nabla} \times \boldsymbol{q}=\mathbf{0}$, then $\boldsymbol{q}=\nabla p$ for some $p \in \tilde{S}_{h}$.

For $\boldsymbol{\Theta} \in \boldsymbol{H}_{0}\left(\operatorname{curl} ; \Omega_{\infty}\right)$, set $\boldsymbol{e}=\boldsymbol{\Theta}-\hat{P}_{h} \boldsymbol{\Theta}$ and let $\boldsymbol{V}_{h}$ be in $Q_{h}$. We decompose $\boldsymbol{e}$ as $\boldsymbol{e}=\boldsymbol{e}_{0}+\boldsymbol{\nabla} \psi$, where $\psi \in \widetilde{H}_{0}^{1}\left(\Omega_{\infty}\right)$ satisfies

$$
(\boldsymbol{\mu} \nabla \psi, \boldsymbol{\nabla} \Phi)=(\boldsymbol{\mu e}, \boldsymbol{\nabla} \Phi), \quad \text { for all } \Phi \in \widetilde{H}_{0}^{1}\left(\Omega_{\infty}\right) .
$$

Note that $\boldsymbol{e}_{0}$ is in $\boldsymbol{X}_{N}\left(\Omega_{\infty}\right)$.

We then decompose $\boldsymbol{V}_{h}$ as $\boldsymbol{V}_{h}=\boldsymbol{V}_{0, h}+\boldsymbol{\nabla} \psi_{h}$, where $\psi_{h} \in \tilde{S}_{h}$ satisfies

$$
\left(\boldsymbol{\mu} \nabla \psi_{h}, \boldsymbol{\nabla} \Phi\right)=\left(\boldsymbol{\mu} \boldsymbol{V}_{h}, \boldsymbol{\nabla} \Phi\right), \quad \text { for all } \Phi \in \tilde{S}_{h} .
$$

Thus, since $\nabla \psi_{h} \in Q_{h}$,

$$
\left(\boldsymbol{\mu} \boldsymbol{V}_{h}, \boldsymbol{e}\right)=\left(\boldsymbol{\mu} \boldsymbol{V}_{0, h}, \boldsymbol{e}\right)+\left(\boldsymbol{\mu} \boldsymbol{\nabla} \psi_{h}, \boldsymbol{e}\right)=\left(\boldsymbol{\mu} \boldsymbol{V}_{0, h}, \boldsymbol{e}\right)+k^{-2} \hat{\mathcal{A}}\left(\boldsymbol{\nabla} \psi_{h}, \boldsymbol{e}\right)=\left(\boldsymbol{\mu} \boldsymbol{V}_{0, h}, \boldsymbol{e}\right) .
$$

Hence

$$
\left(\boldsymbol{\mu} \boldsymbol{V}_{h}, \boldsymbol{e}\right)=\left(\boldsymbol{\mu} \boldsymbol{V}_{0, h}, \boldsymbol{e}_{0}\right)+\left(\boldsymbol{\mu} \boldsymbol{V}_{0, h}, \boldsymbol{\nabla} \psi\right)
$$

We will estimate the two terms in (3.18) separately. To estimate $\left(\boldsymbol{\mu} \boldsymbol{V}_{0, h}, \boldsymbol{e}_{0}\right)$ note that $\left(\boldsymbol{\mu} \boldsymbol{V}_{0, h}, \boldsymbol{V}_{0, h}\right)=\left(\boldsymbol{\mu} \boldsymbol{V}_{0, h}, \boldsymbol{V}_{h}\right)$. Thus, it follows from (3.5) that

$$
\left\|\boldsymbol{V}_{0, h}\right\|_{\boldsymbol{L}^{2}\left(\Omega_{\infty}\right)} \leq C\left\|\boldsymbol{V}_{h}\right\|_{\boldsymbol{L}^{2}\left(\Omega_{\infty}\right)} .
$$

Hence

$$
\left|\left(\boldsymbol{\mu} \boldsymbol{V}_{0, h}, \boldsymbol{e}_{0}\right)\right| \leq C\left\|\boldsymbol{V}_{h}\right\|_{\boldsymbol{L}^{2}\left(\Omega_{\infty}\right)}\left\|\boldsymbol{e}_{0}\right\|_{\boldsymbol{L}^{2}\left(\Omega_{\infty}\right)}
$$


To estimate the right hand side above, we will use a duality argument. The bound for (3.19) is similar to an argument in [10] modified to take into account a multiply connected boundary and complex coefficients appearing from the PML operator.

Let $\boldsymbol{z} \in \boldsymbol{H}_{0}\left(\mathbf{c u r l} ; \Omega_{\infty}\right)$ satisfy

$$
\hat{\mathcal{A}}(\boldsymbol{z}, \boldsymbol{\Phi})=\left(\boldsymbol{\mu} \boldsymbol{e}_{0}, \boldsymbol{\Phi}\right), \quad \text { for all } \boldsymbol{\Phi} \in \boldsymbol{H}_{0}\left(\operatorname{curl} ; \Omega_{\infty}\right) .
$$

Because of (3.6), this problem is well posed. In addition, the solution satisfies $(\boldsymbol{\mu} \boldsymbol{z}, \boldsymbol{\nabla} \theta)=0$ for all $\theta$ in $\widetilde{H}_{0}^{1}\left(\Omega_{\infty}\right)$ and thus $\boldsymbol{z}$ is in $\boldsymbol{X}_{N}\left(\Omega_{\infty}\right)$ so the proposition implies that it is in $\boldsymbol{H}^{s}\left(\Omega_{\infty}\right)$ for some $s>1 / 2$. Also, $\boldsymbol{v}=\boldsymbol{\mu}^{-1} \boldsymbol{\nabla} \times \boldsymbol{z}$ is in $\boldsymbol{X}_{T}\left(\Omega_{\infty}\right)$ and the proposition implies that it, too, is in $\boldsymbol{H}^{s}\left(\Omega_{\infty}\right)$. As $\boldsymbol{\mu}$ is smooth, this implies that $\boldsymbol{\nabla} \times \boldsymbol{z}$ is in $\boldsymbol{H}^{s}\left(\Omega_{\infty}\right)$ and

$$
\|\boldsymbol{z}\|_{\boldsymbol{H}^{s}\left(\Omega_{\infty}\right)}+\|\boldsymbol{\nabla} \times \boldsymbol{z}\|_{\boldsymbol{H}^{s}\left(\Omega_{\infty}\right)} \leq C\left\|\boldsymbol{e}_{0}\right\|_{\boldsymbol{L}^{2}\left(\Omega_{\infty}\right)} .
$$

For any $\boldsymbol{z}_{h} \in Q_{h}$,

$$
\left\|\boldsymbol{e}_{0}\right\|_{\boldsymbol{L}^{2}\left(\Omega_{\infty}\right)}^{2} \leq C\left|\left(\boldsymbol{\mu} \boldsymbol{e}_{0}, \boldsymbol{e}_{0}\right)\right|=C\left|\hat{\mathcal{A}}\left(\boldsymbol{z}, \boldsymbol{e}_{0}\right)\right|=C|\hat{\mathcal{A}}(\boldsymbol{z}, \boldsymbol{e})|=C\left|\hat{\mathcal{A}}\left(\boldsymbol{z}-\boldsymbol{z}_{h}, \boldsymbol{e}\right)\right| .
$$

By the approximation properties of the Nédélec spaces,

$$
\begin{aligned}
\inf _{\boldsymbol{z}_{h} \in Q_{h}}\left|\hat{\mathcal{A}}\left(\boldsymbol{z}-\boldsymbol{z}_{h}, \boldsymbol{e}\right)\right| & \leq C \inf _{\boldsymbol{z}_{h} \in Q_{h}}\left\|\boldsymbol{z}-\boldsymbol{z}_{h}\right\|_{\boldsymbol{H}\left(\operatorname{curl} ; \Omega_{\infty}\right)}\|\boldsymbol{e}\|_{\boldsymbol{H}\left(\operatorname{curl} ; \Omega_{\infty}\right)} \\
& \leq C h^{s}\left\|\boldsymbol{e}_{0}\right\|_{\boldsymbol{L}^{2}\left(\Omega_{\infty}\right)}\|\boldsymbol{e}\|_{\boldsymbol{H}\left(\operatorname{curl} ; \Omega_{\infty}\right) .}
\end{aligned}
$$

Combining the above gives a bound for the first term of (3.18), i.e.,

$$
\left|\left(\boldsymbol{\mu} \boldsymbol{V}_{0, h}, \boldsymbol{e}_{0}\right)\right| \leq C h^{s}\|\boldsymbol{e}\|_{\boldsymbol{H}\left(\operatorname{curl} ; \Omega_{\infty}\right)}\left\|\boldsymbol{V}_{h}\right\|_{\boldsymbol{L}^{2}\left(\Omega_{\infty}\right)} .
$$

We finally bound the last term in (3.18). Let $\Psi \in \widetilde{H}_{0}^{1}\left(\Omega_{\infty}\right)$ solve

$$
(\boldsymbol{\mu} \nabla \Psi, \boldsymbol{\nabla} \theta)=\left(\boldsymbol{\mu} \boldsymbol{V}_{0, h}, \boldsymbol{\nabla} \theta\right), \quad \text { for all } \theta \in \widetilde{H}_{0}^{1}\left(\Omega_{\infty}\right)
$$

and set $\boldsymbol{V}_{0}=\boldsymbol{V}_{0, h}-\nabla \Psi$. Then,

$$
\left(\boldsymbol{\mu} \boldsymbol{V}_{0, h}, \boldsymbol{\nabla} \psi\right)=\left(\boldsymbol{\mu}\left(\boldsymbol{V}_{0, h}-\boldsymbol{V}_{0}\right), \boldsymbol{\nabla} \psi\right), \quad \text { for all } \psi \in \widetilde{H}_{0}^{1}\left(\Omega_{\infty}\right) .
$$

The above proposition shows that $\boldsymbol{V}_{0}$ is in $\boldsymbol{H}^{s}\left(\Omega_{\infty}\right)$.

Let $R_{h}$ denote the corresponding conforming $\boldsymbol{H}_{0}\left(\operatorname{div} ; \Omega_{\infty}\right)$ approximation subspace appearing in the discrete de Rham sequence, e.g., if $Q_{h}$ is the lowest order Nédélec curl-conforming finite element space, $R_{h}$ is the lowest order RaviartThomas div-conforming finite element space. The curl of $\boldsymbol{V}_{0}$ is piecewise polynomial since it coincides with that of $\boldsymbol{V}_{0, h}$ (and hence is in $R_{h}$ ). It follows that the natural interpolation operator $r_{h}$ (onto $Q_{h}$ ) is well defined on $\boldsymbol{V}_{0}$ and satisfies

$$
\left\|\boldsymbol{V}_{0}-r_{h} \boldsymbol{V}_{0}\right\|_{\boldsymbol{L}^{2}\left(\Omega_{\infty}\right)} \leq C\left(h^{s}\left\|\boldsymbol{V}_{0}\right\|_{\boldsymbol{H}^{s}\left(\Omega_{\infty}\right)}+h\left\|\boldsymbol{\nabla} \times \boldsymbol{V}_{0}\right\|_{\boldsymbol{L}^{2}\left(\Omega_{\infty}\right)}\right)
$$

(see, e.g., Theorem 5.41 in [10]).

The natural interpolation operators $r_{h}$ and $\pi_{h}$ for $Q_{h}$ and $R_{h}$, respectively, along with the curl operator satisfy $\pi_{h}(\boldsymbol{\nabla} \times \boldsymbol{u})=\boldsymbol{\nabla} \times\left(r_{h} \boldsymbol{u}\right)$ (for sufficiently smooth $\boldsymbol{u}$ ) from which it follows that

$$
\boldsymbol{\nabla} \times\left(r_{h} \boldsymbol{V}_{0}-\boldsymbol{V}_{0, h}\right)=\mathbf{0} .
$$

The above discussion implies that $r_{h} \boldsymbol{V}_{0}-\boldsymbol{V}_{0, h}=\boldsymbol{\nabla} \phi_{h}$ for some $\phi_{h} \in \tilde{S}_{h}$. It follows that $\left(\boldsymbol{\mu}\left(\boldsymbol{V}_{0}-\boldsymbol{V}_{0, h}\right), r_{h} \boldsymbol{V}_{0}-\boldsymbol{V}_{0, h}\right)=0$. Hence

$$
\left(\boldsymbol{\mu}\left(\boldsymbol{V}_{0}-\boldsymbol{V}_{0, h}\right), \boldsymbol{V}_{0}-\boldsymbol{V}_{0, h}\right)=\left(\boldsymbol{\mu}\left(\boldsymbol{V}_{0}-\boldsymbol{V}_{0, h}\right), \boldsymbol{V}_{0}-r_{h} \boldsymbol{V}_{0}\right) .
$$


The estimate

$$
\begin{aligned}
\left\|\boldsymbol{V}_{0}-\boldsymbol{V}_{0, h}\right\|_{\boldsymbol{L}^{2}\left(\Omega_{\infty}\right)} & \leq C\left\|\boldsymbol{V}_{0}-r_{h} \boldsymbol{V}_{0}\right\|_{\boldsymbol{L}^{2}\left(\Omega_{\infty}\right)} \leq C h^{s}\left\|\boldsymbol{V}_{0}\right\|_{\boldsymbol{H}\left(\mathbf{c u r l} ; \Omega_{\infty}\right)} \\
& \leq C h^{s}\left\|\boldsymbol{V}_{h}\right\|_{\boldsymbol{H}\left(\mathbf{\operatorname { c u r l }} ; \Omega_{\infty}\right)}
\end{aligned}
$$

follows easily. Combining the above gives

$$
\begin{aligned}
\left(\boldsymbol{\mu} \boldsymbol{V}_{0, h}, \boldsymbol{\nabla} \psi\right) & \leq C h^{s}\left\|\boldsymbol{V}_{h}\right\|_{\boldsymbol{H}\left(\operatorname{curl} ; \Omega_{\infty}\right)}\|\boldsymbol{\nabla} \psi\|_{\boldsymbol{L}^{2}\left(\Omega_{\infty}\right)} \\
& \leq C h^{s}\left\|\boldsymbol{V}_{h}\right\|_{\boldsymbol{H}\left(\operatorname{curl} ; \Omega_{\infty}\right)}\|\boldsymbol{e}\|_{\boldsymbol{L}^{2}\left(\Omega_{\infty}\right)} .
\end{aligned}
$$

This gives the desired bound for the second term of (3.18) and completes the proof of the lemma.

\section{REFERENCES}

[1] C. Amrouche, C. Bernardi, M. Dauge, and V. Girault. Vector potentials in three-dimensional non-smooth domains. Math. Methods Appl. Sci., 21(9):823-864, 1998. MR1626990 (99e:35037)

[2] G. Bao and H. Wu. Convergence analysis of the perfectly matched layer problems for timeharmonic Maxwell's equations. SIAM J. Numer. Anal., 43(5):2121-2143 (electronic), 2005. MR 2192334

[3] J. H. Bramble and J. E. Pasciak. Analysis of a finite PML approximation for the three dimensional time-harmonic maxwell and acoustic scattering problems. Math. Comp., 76 (2007), 597-614.

[4] F. Collino and P. Monk. The perfectly matched layer in curvilinear coordinates. SIAM J. Sci. Comp., 19(6):2061-2090, 1998. MR 1638033 (99e:78029)

[5] V. Girault and P. Raviart. Finite Element Approximation of the Navier-Stokes Equations. Lecture Notes in Math. 749, Springer-Verlag, New York, 1979. MR0548867 (83b:65122)

[6] J. Gopalakrishnan and J. E. Pasciak. Overlapping Schwarz preconditioners for indefinite time harmonic Maxwell equations. Math. Comp., 72(241):1-15, 2003. MR.1933811(2003i:78020)

[7] F. Kukuchi. On a discrete compactness property for the Nédélec finite elements. J. Fac. Sci. Univ. Tokyo, Sect. 1A, Math, 36:479-490, 1989. MR1039483 (91h:65173)

[8] M. Lassas and E. Somersalo. On the existence and convergence of the solution of PML equations. Computing, 60(3):229-241, 1998. MR1621305 (99a:65133)

[9] P. Monk. A simple proof of convergence for an edge element discretization of maxwell's equations. Lecture Notes in Comp. Sci. Engl., 28, Springer, Berlin, 2003. MR1986135 (2004i:78024)

[10] P. Monk. Finite element methods for Maxwell's equations. Oxford Science Pub., Oxford, 2003.

[11] P. Monk and L. Demkowicz. Discrete compactness and the approximation of Maxwell's equations in $\mathbb{R}^{3}$. Math. Comp., 70(234):507-523, 2001. MR:1709155 (2001g:65156)

[12] J. C. Nédélec. Mixed finite elements in $\mathbf{R}^{3}$. Numer. Math., 35:315-341, 1980. MR592160 (81k:65125)

[13] J. C. Nédélec. A new family of mixed finite elements in $\mathbf{R}^{3}$. Numer. Math., 50:57-81, 1986. MR864305 (88e:65145)

[14] A. Schatz. An observation concerning Ritz-Galerkin methods with indefinite bilinear forms. Math. Comp., 28:959-962, 1974. MR0373326 (51:9526)

Department of Mathematics, Texas A\&M University, College Station, Texas 778433368.

E-mail address: bramble@math.tamu.edu

Department of Mathematics, Texas A\&M University, College Station, Texas 778433368.

E-mail address: pasciak@math.tamu.edu 